\title{
Mario Vargas Llosa y sus detectives frustrados
}

\author{
Dr. Clemens A. Franken Kurzen*
}

\section{Resumen:}

Luego de exponer los antecedentes bio-bibliográficos más relevantes, la visión de la sociedad y el concepto de la literatura y del lector del escritor peruano Mario Vargas Llosa se analiza en forma detallada su novela con formato policial ¿Quién mató a Palomino Molero? (1986), señalando rasgos característicos de los detectives, su forma de pensar, su perfil psicológico y su modo de investigar, los motivos sociales y culturales del crimen y la crítica a la sociedad peruana. Especial énfasis se pone en destacar la hibridez inter e intragenérica presente en esta obra literaria, es decir, la mezcla de diferentes géneros literarios y subgéneros policiales.

Palabras clave: novela policial latinoamericana - asimilación híbrida - novela policial

\section{Abstract:}

After presenting his most relevant bio-bibliographic antecedents, his view of society and his concept of literature, Mario Vargas Llosa's novel Who killed Palomino Molero? (1986), written with a detective fiction format, is analysed in detail pointing at characteristic features that detectives have, at their way of thinking, at their psychological profile and at their method of investigation; the social and cultural motives for crime and a criticism towards Peruvian society are also analysed. Special attention is paid to highlighting the inter and intrageneric hybridation -the mixture of different literary genres and detective subgenres- that appear in this novel.

Keywords: Detective Fiction from Latinamerica - hybrid assimilation

* Doctor en Literatura. Académico de la Pontificia Universidad Católica de Chile. cfranken@uc.cl.

Este artículo es fruto del Proyecto Fondecyt Regular № 1071100 “Hibridaciones, parodias y polémicas. con el género policial en la narrativa latinoamericana". 


\section{Objetivo del artículo}

El objetivo de este artículo es corroborar que Vargas Llosa, al igual que, por ejemplo, García Márquez con Crónica de una muerte anunciada o Soriano con Triste, solitario y final, escribe con ¿Quién mató Palomino Molero? una novela policial híbrida inter-e intragenérica, mezclando tanto diferentes géneros literarios como subgéneros policiales.

\section{Antecedentes bio-bibliográficos}

Mario Vargas Llosa, sin duda uno de los escritores latinoamericanos más relevantes, nace en Arequipa el año 1936 "en el seno de una familia relativamente acomodada y de rancio apellido. Al separarse sus padres, poco después de su nacimiento, creció en la casa de sus abuelos maternos. Es el niño consentido por excelencia" (Castro 22). Junto a su abuelo materno diplomático pasa ocho años de su infancia en Cochabamba (Bolivia) y luego cinco años en Piura, donde es matriculado en un colegio particular religioso. Con catorce años recibe el primer gran golpe de su vida cuando su padre lo hace "entrar al Colegio Militar Leoncio Prado porque en [su] opinión [...] había que corregir al engreído y afeminado muchacho que le había entregado la familia de su esposa" (22). En sus memorias tituladas El pez en el agua, Vargas Llosa recuerda las profundas huellas que dejó en él esta decisión paternal: “En los años que viví con mi padre [...] se desvaneció la inocencia, la visión candorosa que mi madre, mis abuelos y mis tíos me habían inculcado. En esos tres años descubrí la crueldad, el miedo, el rencor" (101). Los dos años siguientes en el colegio militar lo marcan para siempre. A pesar de que su padre se opuso tempranamente a la vocación literaria de su hijo, Vargas Llosa estudia Letras en la Universidad de San Marcos y se inicia en el ejercicio del periodismo cuando aún era un estudiante de bachiller, colaborando en los periódicos La Crónica y La Industria. Poco después entabla una relación amorosa con su tía política, Julia Urquidi, con quien se casa en 1955 y viajan juntos a 
Europa. Aunque ya con anterioridad había escrito una obra de teatro, en 1957 comienza a publicar cuentos en algunas revistas: "Los jefes" aparecerá en El Mercurio Peruano y "El abuelo" en El Comercio.

En 1958 viaja a París, gracias a un premio recibido en un concurso de relatos, y a su regreso a Lima recibe una beca para trasladarse a la Universidad de Madrid. A los pocos meses abandona los estudios de doctorado y fija su residencia en París, donde permanecerá durante siete años.

En 1963 se publicará su primera gran novela, La ciudad y los perros, que obtiene varios premios literarios y lo consagra como novelista. A esta primera novela le siguen una abundante producción del mismo género: La casa verde (1966), Conversación en la catedral (1969), Pantaleón y las visitadoras (1973), La tía Julia y el escribidor (1977), La guerra del fin del mundo (1981), Historia de Mayta (1984), ¿Quién mató a Palomino Molero? (1986), El hablador (1987), Elogio de la madrastra (1988), Lituma en los Andes (1993), Los cuadernos de don Rigoberto (1995), La fiesta del chivo (2000), El paraíso en la otra esquina (2003) y, últimamente, Travesuras de la niña mala (2006).

Además de estas novelas, que confirman por si solas su característica "fuerza de voluntad y perseverancia", como su también "insistencia en la vocación y el trabajo duro" (Angvik 25), Vargas Llosa escribió también cuentos, siete obras teatrales, un sinnúmero de ensayos y memorias, que junto con sus novelas conforman una gran e impresionante obra literaria.

Sin embargo, hay otra vertiente en la vida de este autor peruano que siempre ha despertado interés: la política. Incluso en el año 1987 se lanza en una candidatura presidencial por el Frente Democrático de tendencia liberal y conservadora. Esto le valió el reproche de muchos escritores e intelectuales, puesto que en su juventud Vargas Llosa tenía filiación con el Partido Comunista, del cual se alejó luego de su públicamente manifestado desencantamiento de la Revolución Cubana, a diferencia de su ex amigo Gabriel García Márquez quien continúa hasta la actualidad apoyando a Fidel Castro. Dado que Vargas Llosa pierde contra Fujimori en las elecciones presidenciales del 1990 se retira de la arena política y opta por realizar su aporte a la sociedad peruana exclusivamente desde la literatura.

\section{Visión de la sociedad peruana}

La visión de país que Vargas Llosa recalca permanentemente en su obra literaria es la "de una sociedad dividida por abismos geográficos, 
sociales y, particularmente, raciales. Él ve a un país fragmentado por múltiples antagonismos y poderosas barreras. Quienes osan cruzar estas barreras, sólo consiguen desarraigarse" (Brown 23). Esta realidad caótica ha sido llamada por otros intelectuales como un "political, social, and emotional labyrinth", del cual no hay escapatoria, dado que en el "nothing really changes" (Gallagher 125 y 133).

Ahora bien, los rasgos característicos de esta caótica sociedad peruana son, según Vargas Llosa, ante todo, el machismo y el racismo. Para D.G. Gallagher, el machismo o la aserción de la masculinidad es una "very Latin American condition", que se hace presente, por ejemplo en la novela $L a$ ciudad y los perros, en el personaje de la Malpapeada, quien en presencia de sus amigos "must sustain an image that is based on his principal claim to fame, that he has the largest sexual organ in the school. Machismo is the young boy's passport to mutual acceptance" (131). Esta categórica afirmación es recalcada por el crítico literario alemán Thomas Scheerer, según el cual "la sexualidad es el impulso básico que revela los lados malos del actuar humano" (135). ${ }^{1}$

Respecto al otro rasgo propio de la sociedad peruana, el racismo, James W. Brown destaca el hecho de que en las primeras tres novelas de Vargas Llosa "la raza y el racismo juegan un papel importante" y distingue cinco diferentes "aglomeraciones raciales del Perú": 1) algunos personajes se refieren a "la población blanca, predominantemente costera o criolla, con el nombre de blanquiñosos, término despectivo utilizado en replana, o sea, la jerga costera"; 2) "los cholos, término que se refiere a las personas cuya apariencia y/o hábitos de vida lo marcan racialmente como indígena"; 3) En un peldaño inferior de la escala racial se encuentran "los serranos, que pertenecen a la estirpe indígena de los Andes, como los cholos, pero que están menos integrados aún en la sociedad criolla"; 4) Peor tratados son aún "los negros y los zambos, términos que los personajes de Vargas Llosa utilizan indistintamente para referirse a los negros"; y 5) los menos apreciados y más apartados de la sociedad criolla son "los indios amazónicos", las cuales son considerados "como subhumanos e incluso como animales" (Brown 15-16).

Ahora bien, las grandes instituciones que sostienen esta realidad caótica marcada por el machismo y racismo son el ejército, la policía, la dictadura y la Iglesia Católica. Comenzando con la última, Vargas Llosa le reprocha, ante todo, su fanatismo e intolerancia (cfr. Oviedo, 1981:

"die Sexualität der eigentliche Antrieb für die bösen Seiten menschlichen Handelns ist." Luego Scheerer cita parte de una recensión que Vargas Llosa hace sobre la obra de William Faulkner, donde sostiene que la sexualidad no hace feliz a las figuras del autor norteamericano, ni facilita la comunicación, ni consolida la solidaridad. Casi siempre es una experiencia que degrada y destruye al hombre convirtiéndolo en animal (cfr. 135). 
53). Más dura y extensa es la crítica a los militares que en cierta medida incluye a la policía y a la dictadura:

En primer lugar, el mundo militar se le aparece una maquinaria de naturaleza cerrada, con sus códigos secretos y autosuficientes, casi una francamasonería que se funda en símbolos, valores y propósitos que el resto de la sociedad no comparte o no conoce por completo. El sistema militar se reviste así con un aura de prestigio ante los ojos de los individuos e instituciones civiles, cuya principal carencia es la falta de unidad y cohesión interna, la tendencia a la descomposición. (Oviedo, 1981:53)

En la novela La ciudad y los perros, por ejemplo, el Jaguar "enuncia la esencia de esta ley [militar] con brutal precisión: 'En el colegio todos friegan a todos, el que se deja se arruina. No es mi culpa. Si a mí no me joden es porque soy más hombre'"' (Oviedo, 1981: 50).

Esta visión negativa de las grandes instituciones del Perú hace comprender que en las novelas de Vargas Llosa siempre existen individuos que "entren en pugna con las jerarquías (militares, políticas, religiosas), que intentan desconocer sus reglas y explorar por sí mismos el tentador mundo de fuera. [...]; generalmente son destruidos [...], o son tristemente reabsorbidos por la jerarquía, degradados por la doble abdicación" (Oviedo, 1981: 53-54).

Mario Vargas Llosa sufrió en carne propia estas imposiciones y jerarquías y se alejó conscientemente de su clase social que consideraba repugnante por todos los aspectos antes indicados y se retiró tempranamente a un exilio voluntario en Europa donde continuamente escribió sobre su país de origen "a través de los recuerdos de su infancia y juventud. [Vargas Llosa] ha confesado que el acto de escribir es, para él, producto de una inexorable insatisfacción y extrañamiento, [...]" (Brown 24). ${ }^{2}$

\section{Concepto de la literatura y del lector e influencias}

La mala realidad peruana e, incluso, latinoamericana, con sus injusticias, sus explotaciones, su desigualdad, su pobreza, su alienación cultural y moral no deja, según él, otra alternativa que rebelarse contra ella (cfr. La literatura es fuego 20). Escribir significa para Vargas Llosa, por

2 Según Efraín Kristal, el joven socialista Mario Vargas Llosa creía, siguiendo a J.P. Sartre, que esta insatisfacción vendría de la naturaleza del orden capitalista injusto, mientras que el escritor más maduro y neoliberal la ubica más bien en la naturaleza humana (cfr. XIV). 
eso, rebelarse contra esta realidad insatisfactoria recreándola a través de la realidad ficticia que es una suprarrealidad. Como un ser marginado, extravagante, excéntrico y angustiado, el escritor se libera, según él, de sus demonios, obsesiones, fantasmas y rencores a través de la escritura que él describe como un 'estriptease intelectual'. Fuentes de la narrativa son para él los demonios personales, históricos y culturales, es decir la experiencia personal de cada escritor que le da a su obra un claro carácter autobiográfico.

Esto se relaciona con el hecho de que la ficción suele surgir, ante todo, en períodos históricos conflictivos, en que las bases y principios del orden social y del sentido de la vida se encuentran en crisis. Según el mismo Vargas Llosa, el género literario de la novela toma auge en los períodos finales de la Edad Media. Esto debido a que la razón intentó sin éxito suplantar a Dios, por lo que se perdió la confianza en el orden del universo que hasta ese entonces respaldaba la fe, y se comenzó a mirar al mundo y a la vida como una confusión caótica, provocando un vacío que necesitaba ser llenado por algún principio sustitutivo. Justamente el novelista se adelanta en percibir el debilitamiento de la fe en el orden establecido y se convierte en "suplantador de Dios" o "deicida" o "segundo Dios" al rebelarse, por una parte, contra el mundo creado por Dios cuyas deficiencias, injusticias y problemas rechaza, como por otra parte, al crear un nuevo orden literario del mundo percibido como caótico. La tarea del escritor es justamente ordenar este para facilitarle al lector así la comprensión de esta "realidad heterogénea, contradictoria y compleja, que solo puede ser expresada barajando otros niveles que los establecidos por la narrativa tradicional realista, [...], [mezclando] lo fantástico y lo cotidiano, lo mágico y lo épico y lo social y lo religioso y lo político y lo sentimental..." (Osorio 71). De esta forma, "la escritura es una búsqueda de las raíces de la insatisfacción y un intento de reintegrarse a la sociedad de la cual se siente excluido" (Angvik 22). La búsqueda de la realidad social e histórica se convierte de esta forma en una característica destacada de la novelística de este autor peruano. En esta búsqueda de la realidad peruana y de su propia identidad Vargas Llosa se basa en datos objetivos sociológicos, psicológicos, históricos y antropológicos, sin embargo, "'los traslada al espacio de los mitos secularizados, que manejan la conducta de los hombres. En eso se sirve de todos los niveles de interpretación de las 'formas simples' modernas desde los romances de la literatura trivial, pasando por el western hasta llegar a la película de amor sentimental de procedencia mexicana'"' (Oviedo citado en Kloepfer /Zimmermann 484). ${ }^{3}$ Sarah Castro agrega al respecto que tanto la novela Historia de Mayta como ¿Quién mató Palomino Molero? "comparten con las radionovelas

"als resistent gegenüber der ermittelten Wahrheit und damit als gesellschaftlich wirkungsvoller" 
muchos elementos de la producción de intrigas" (130) y destaca luego la cercanía del quehacer novelístico de Vargas LLosa con el del "periodista investigador" (131): ambos buscan la verdad histórica y tienen, además, "fábulas organizadas por medio de una línea cronológica que mantiene un suspenso más o menos interesante" (136). Esta búsqueda de la verdad social e histórica es siempre un gran desafío para un narrador-descubridor ya sin ideales revolucionarios y para quien "ultimately nothing is certain" (Gallagher 141).

A este narrador-descubridor corresponde un lector que se hace "cómplice en la tarea de exorcizar la realidad" (Osorio, 1970: 88) y que desea reconstruir junto con el narrador la historia del Perú y de América Latina descubriendo las verdades ocultas y desenmascarando los vicios de la sociedad. Vargas Llosa postula, por eso, una participación activa del lector en el proceso de la lectura. El novelista debe suponer y respetar la libertad del lector apelando a ella para estimularlo a interiorizarse en su obra. De esta manera, según los principios teóricos de la Estética de la recepción, la novela moderna desafía al lector, le propone arriesgarse a una aventura detectivesca e interpretativa que le demande el sagaz ejercicio de su ingenio y percepción. Vargas Llosa se revela en este punto, además, como fiel admirador de Jean Paul Sartre quien percibió la literatura como un encuentro de dos libertades y quien dijo que no se escribe para esclavos.

Otro escritor importante para Vargas Llosa es el novelista francés G. Flaubert a quien considera el iniciador de la tendencia literaria de borrar de la novela la huella del autor, desterrando la omnisciencia del narrador clásico y garantizando de esta forma la existencia de tantas interpretaciones como lectores se aboquen a su lectura. Vargas Llosa hizo suya esta aspiración de crear mundos justificados por sí mismos, independientes de toda presión ajena e influencia extraña, debiendo quedar oculta en la mayor medida posible toda señal de la paternidad de la obra. En su afán de abarcar toda la realidad incorpora a su descripción del mundo exterior la búsqueda en zonas más oscuras, pero no por eso menos verdaderas, como los sueños, las fantasías, obsesiones y delirios de la conciencia. Su mundo poético se organiza como un universo coherente, cuyos principios rectores se mantienen en cada una de las obras individuales, donde cada elemento adquiere un significado que espera ser descubierto. Este carácter connotativo de los pilares de su obra demanda del lector una actitud de desconfianza y recelo frente al narrador que lo engaña con expectativas falsas, haciéndolo tropezar una y otra vez durante la lectura para obligarlo a desarrollar su propia capacidad en búsqueda de las verdades ocultas. En las novelas de Vargas Llosa no aparecen guías omniscientes, dueños de las criaturas y del mundo, sino descubridores que invitan al lector a asumir como cómplice la aventura 


\section{Análisis de ¿Quién mató a Palomino Molero?}

Para contextualizar bien la novela ¿Quién mató a Palomino Molero?, hay que considerar que Vargas Llosa enfrenta en ella dos desafíos. En primer lugar, en esta novela juega un papel importante el intento de mostrar sus dudas respecto a la posibilidad de dar con la verdad social e histórica que ya vimos presentes en la novela anterior Historia de Mayta (cfr. Scheerer 204). Según Efraín Kristal, tras finalizar la recién nombrada novela y antes de escribir ¿Quién mató a Palomino Molero?, Vargas Llosa "agreed to become a member of the 'Investigatory Commission' that inquired into the brutal murder of eight journalists by the villagers of Uchuraccay in January 1983" (150). Su participación en esta comisión investigadora fue interpretada como un intento de nuestro escritor de apoyar y legitimar al gobierno peruano de aquel entonces, lo que le ha causado el surgimiento de difamaciones, polémicas, y estudios especializados por parte de académicos. Por eso, algunos críticos sostienen que esta novela puede ser leída "as a literary exorcism of his own experiences on the commissión" (Kristal 151). Tanto Vargas Llosa en la vida real peruana como los investigadores Silva y Lituma en la novela ¿Quién mató a Palomino Molero? deben enfrentar la incredulidad de la gente respecto a la verdad que descubrieron. Los prejuicios de la gente se muestran en ambos casos "como resistentes frente a la verdad investigada y con eso como socialmente más efectivos" (Scheerer136). ${ }^{4}$

En segundo lugar, esta novela significa para nuestro autor un nuevo y gran desafío formal. Sabemos que esta novela surgió del deseo de Vargas Llosa de superar su tendencia de referirse en forma extensa a todos los aspectos y elementos de la realidad y en este sentido se acerca a posturas borgeanas de escribir en forma más racional y más concentrado en lo esencial, haciendo uso consciente de todos los medios técnicos de la

4 “'sie aber überführt in den Raum säkularisierter Mythen, die das Verhalten der Menschen steuern. Dabei benutzt er alle Interpretationsraster der modernen 'einfachen Formen' von den Romanzen der Trivialliteratur, über die Westernstory bis hin zum sentimentalen Liebesfilm mexikanischer Prägung".

Según el crítico literario alemán Thomas Scheerer, luego de las tres primeras grandes novelas en los años sesenta, Vargas Llosa se nos presenta cada vez de "una 'nueva manera'" ("eine 'neue Manier'") (132), creando diferentes tipos de novelas híbridas. Él justifica su modus procedendi por el deseo de no querer repetirse y de necesitar nuevos desafíos formales. 
novela policial. Según él, la novela ¿Quién mató Palomino Molero? "no contiene ningún elemento que sobre" (citado en Scheerer 133). ${ }^{5}$

De esta forma, el joven y rebelde escritor-descubridor-desenmascarador siente el desafío de convertirse cada vez más en un escritor "Artifex", es decir, en un artista planificador.

La novela comienza con el hallazgo de un cadáver colgado de un árbol y gravemente vejado. El guardia del puesto de policía de Talara, Lituma, señala en el lugar de los hechos que no hay duda de que se trata de un homicidio y su acompañante, el taxista don Jerónimo, reconoce en el muerto "al piuranito que cantaba boleros" (PM 8). Más tarde, en un diálogo de los 'inconquistables', se confirma la identidad del muerto: Palomino Molero, un recluto de la Base Aérea de Talara.

Sin haber sido asignado al caso, Lituma emprende por su cuenta las averiguaciones no sin hacernos saber su temor por las consecuencias que esto pudiera causarle. Luego también y casi extraoficialmente, dado que se trata de un asunto que involucra a un miembro de una institución con fueros especiales, se involucra el teniente Silva que prontamente toma la iniciativa pasando a ser Lituma una especie de interlocutor sentimental de la lógica de su superior. Ambas figuras nos son conocidas como personajes flotantes en la obra literaria de Vargas Llosa. Primero aparecen juntos en el escenario de la ciudad de Piura en el cuento "Un visitante" en Los jefes y luego investigan juntos en la Historia de Mayta. Además, en la novela La casa verde y en la obra teatral La Chunga, Lituma es uno de los campeones que frecuentaban el bar de la Chunga. Finalmente este personaje investiga asesinatos y desapariciones atribuidas al Sendero Luminoso en la novela Lituma en los Andes, a la cual me referiré en otra ocasión.

En nuestra novela en cuestión, ambos investigadores saben que pisan un terreno peligroso y que seguramente no contarán con la simpatía de la superioridad policial, pero se ha hecho un deber para ellos terminar la investigación hasta las últimas consecuencias y dar con los culpables de tan horrendo crimen.

Ambos descubren que Palomino Molero era un humilde jovencito del poblado de Piura enamorado de Alicia Mindreau, hija del coronel de la Base Aérea del lugar. Tocaba la guitarra y cantaba cada noche serenatas a su amada. Tras el traslado del coronel a la base de Talara, no le quedó más remedio a Palomino que enrolarse como avionero para estar cerca de Alicia. Desde que el padre tuvo noticia de ello, prohibió a

"kein Element enhält, das überflüssig wäre" 
su hija continuar con el romance. Palomino y Alicia se fugan al pueblo de Amatope en busca de un cura que los case, pero lamentablemente éste no se encuentra en esos momentos. Tras la espera de dos días en una pensión, son descubiertos por el coronel y por el alférez Dufó, novio oficial de Alicia, y llevados de vuelta a Talara. Los demás hechos narrados corresponden a las versiones a menudo contradictorias de los personajes implicados en el caso.

A pesar de que Vargas Llosa, ante todo al final de la novela, intenta enigmatizar la realidad, haciendo bastante verosímil hasta tres versiones distintas de los hechos, las pistas de Alicia Mindreau, las mismas palabras del alto oficial Mindreau en la última entrevista con Silva y Lituma y, finalmente, el tiro que resuena en la noche nos dan suficientes indicios de que el culpable era el coronel Mindreau a quien conocemos ya desde las primeras páginas del segundo capítulo de la novela.

Según Alicia, su padre además de abusar de ella, quería evitar que anduviera con un pobre avionero de inferior condición social, y por esa causa lo mandó matar. El coronel, por su parte, sostiene que su hija padecía de alucinaciones y que todo lo dicho por ella era invento y por eso había querido evitar que Palomino se casara con ella.

Aunque Silva y Lituma no sabían si Alicia era enferma o no, estaban seguros que el coronel era por lo menos el autor intelectual del crimen y que el alférez Dufó, según el cual Palomino Molero se metió en círculos que no le correspondían y por eso mereció que lo mataran, fue probablemente el ejecutor del crimen. Los rumores que circulaban al final de la novela respecto a que Palomino habría estado involucrado en contrabando o que habría descubierto un espionaje y habría sido muerto para que no hablara, por un lado, convierten la investigación de Silva y Lituma en solamente "partially successful" (Kristal 152) y, por orto lado, crean cierta ambigüedad y hacen tal vez posible negar la existencia de una versión objetiva de los hechos, aunque no alcanzan a borrar por completo la validez de los indicios antes mencionados.

Sin embargo, si agregamos a esta cierta enigmatización de la realidad, que significa al mismo tiempo un cierto fracaso de los detectives Silva y Lituma, el fracaso del teniente Silva en el plot paralelo, es decir, en su conquista amorosa de Doña Adriana, - según Scheerer (cfr. 134) una comedia comparable a las de la tradición realista de Cervantes -, y el hecho que ambos son al final castigados y relegados por las autoridades policiales superiores, podemos hablar con cierta razón de "una historia anti-detectivesca" (Badenberg/Honold/Horstmann 286), donde se parodia tanto las averiguaciones policiales como los intentos amorosos. Digo con cierta razón, porque aquí no estamos frente a una nítida inversión de la trama 
tradicional sino frente a un género policial híbrido que contiene tanto elementos de la novela de enigma como de la novela negra. Hay análisis, misterio, suspenso y acción propios del género policial. En comparación con otras novelas policiales latinoamericanas, ésta se acerca más a la de G. García Márquez, ante todo por el común trasfondo socio-histórico y cultural de América Latina y el carácter de novela policial híbrida.

Según Badenberg/Honold/Horstmann (cfr. 282ss), la estructura o la composición de esta novela corresponde a la tradicional tríada de acción: 1.- exposición del asesinato; 2.- proceso de averiguación y reconstrucción y 3.- solución del caso. El primer capítulo cuenta el hecho del asesinato, describiendo el caso y el lugar, introduciendo a la víctima, Palomino Molero, y al personaje de identificación, el guardia Lituma. Los capítulos dos a seis representan la fase de la reconstrucción con los interrogatorios de todas las personas vinculadas al asunto, una por cada capítulo: la madre de Palomino, Doña Asunta; el coronel Mindreau, su jefe; el oficial Ricardo Dufó, el novio de la hija de Mindreau; Doña Lupe, la dueña de la casa donde Palomino y su amante Alicia Mindreau buscaron refugio en su fuga; $y$, por fin, Alicia Mindreau, que termina la fase de la reconstrucción revelando su secreto. En el séptimo capítulo tenemos la parte final de la novela policial clásica: la solución del enigma y la confesión del autor, el coronel Mindreau, quien prueba su culpa al suicidarse y dejar una carta con la confesión de que mató también a su hija. El capítulo ocho funcionaría en este esquema como epílogo, refiriéndose a las versiones del pueblo y el final de la historia personal del teniente Silva.

Entre los personajes, el guardia Lituma tiene para el lector el primer rol en esta novela. El es la única persona que está presente en todas las escenas y toda la historia está descrita desde su perspectiva. Como cholo, Lituma es el subordinado del teniente Silva y procede de un barrio popular de Piura, al igual que Palomino Molero. Luego de una vida más bien inestable y desordenada se hizo guardia debido al salario y a las comidas regulares que este trabajo le ofrecía. El cruel asesinato de Palomino Molero lo impresiona tremendamente por dos razones: en primer lugar por el carácter sexual del mismo, ya que el cadáver fue encontrado castrado y violado ("los huevos le colgaban hasta la entrepierna" (PM 5). A pesar de que Lituma poseía generalmente bastante interés por todo lo relacionado con el sexo y siendo él a su vez un cliente regular de prostíbulos, el carácter sexual tan fuerte de este asesinato parece casi amenazarlo personalmente. En segundo lugar, le fascina y casi obsesiona como miembro de la clase baja peruana, la transgresión de las fronteras entre las clases sociales presente en esta historia policial.

Sin embargo, a Lituma no le mueve un interés analítico como correspondería a un buen guardia que además asume el rol de detective 
en una novela policial, sino que él admira a su superior, al teniente Silva, sus pensamientos geniales, sus técnicas de interrogación. Al igual que el narrador en los cuentos policiales de Poe y, ante todo, a Watson en los de Arthur Conan Doyle, Lituma tiene varias funciones: ser el ayudante del detective extraordinario, transmitir al lector su admiración por su jefe genial, dialogizar la narración y forzar finalmente a revelar un poco su pensamiento frente al narrador y al lector. Al igual que Watson, es menos racional y frío que su jefe $y$, ante todo, le toca hacer conjeturas apresuradas y por eso equivocadas.

El teniente Silva, al igual que Auguste Dupin y Sherlock Holmes, es un personaje algo extravagante con pequeñas imperfecciones y manías, posee una inteligencia superior y cuenta con métodos muy sutiles. Parece tener algunos rasgos "occidentales" como por ejemplo un "bigotito rubio" y una tez "blanquiñosa". Es, además, algo presumido. Su método de investigación, además de conversaciones casuales, entrevistas formales y no pocos hechos fortuitos (cfr. Kristal 154), está basado en la desencantadora y posmoderna experiencia personal de nuestro autor en la ya mencionada investigación en Uchuraccay de 1983 que encuentra su expresión verbal en la sentencia del personaje ficcional de Silva de que "[l]as verdades que parecen más verdades, si les das muchas vueltas, si las miras de cerquita, lo son sólo a medias o dejan de serlo" (PM 107). Según Kristal, Vargas Llosa usa la teoría de la falsificación de Karl Popper en ¿Quién mató a Palomino Molero? "by developing conjectures that collapse like castle of cards [-...-] when the facts refute them allow the two police officers do develop new conjectures" (153). A diferencia de Lituma, Silva no elabora conjeturas precipitadas, sino al estilo neopositivista popperiano procede en forma muy cautelosa elaborando nuevas hipótesis solamente después de que se falsificó la anterior. Un ejemplo: después de interrogar a Doña Lupe en Amotape, Lituma conjetura que el coronel Mindreau y el teniente Dufó mataron a Palomino Molero y que el caso está cerrado. Sin embargo, Silva le da una lección popperiana al decirle que a él solo le consta que dos hombres vinieron acá y se llevaron a los novios. Luego Lituma propone una nueva conjetura con la cual Silva está de acuerdo: Palomino se enroló en la Base Aérea para estar cerca de Alicia (cfr. Kristal 153).

El honesto e íntegro teniente Silva aparece caracterizado en esta novela, ante todo, por su única debilidad: su fijación sexual con Doña Adriana, una respetable mujer casada con un pescador y madre de hijos ya mayores que muy bien podría ser la madre del teniente. Ella es la dueña de la fonda donde los dos policías siempre comen y representa la opinión pública. Según Badenberg/Honold/Horstmann, "el episodio del Teniente y la 'gordita' además está citando las tradiciones literarias de otro género", porque Silva "hace alusiones a las novelas de caballería andante" (284) 
que le son tan queridas para Vargas Llosa. De hecho, Silva espera que Doña Adriana le "premiará con una nochecita" si él logra encontrar los criminales y ponerlos "esposados a sus pies" (PM 27). ${ }^{6}$

Siguiendo con el análisis de los personajes, el coronel Mindreau, por su parte, muestra en las entrevistas que Silva y Lituma sostienen con él un comportamiento arrogante, altanero, lleno de prejuicios sociales y raciales. La relación con su hija tiene un carácter emocional muy intenso y perverso; él la ha educado sin el apoyo de la madre lo que 'explica' la relación incestuosa. Luego de confesar a los detectives su anterior orden de matar a Palomino Molero no manifiesta remordimiento alguno.

Su hija Alicia Mindreau lo odia y parece estar por encima de los prejuicios sociales y raciales de su padre, pues se enamoró de un cholo. Sin embargo, al final de la novela, en sus declaraciones al teniente Silva, cuando dice de su enamorado que "[t]enía el pelo finito y hasta rubio" y que "lo único que tenía de cholo era el nombre ese, Palomino" (PM 123), revela que en el fondo no acepta "ni su condición social ni su raza" y se muestra como una típica "hija mimada de la clase alta" (Badenberg/ Honold/Horstmann 296).

La división de la sociedad peruana en dos clases y diferentes razas se muestra, ahora, tanto en la existencia de dos espacios (la base aérea y la compañía de petróleo) herméticamente separados e inaccesibles para la gente del pueblo como en el uso del lenguaje que despierta especial interés en esta novela policial (cfr. 288 y 297). En primer lugar, el lenguaje utilizado y el modo de expresión manifiestan claramente la jerarquía de poderes y los roles asociados a las diferentes clases sociales. Por ejemplo, en el interrogatorio al coronel Mindreau, éste "murmura con urbanidad", "ataja" a Silva y Lituma, "lee con voz átona", etc., mientras que un Silva intimidado "repusa con mucho respeto" y "sonríe" al coronel. Lituma incluso "balbucea" y "susurra" solamente. De manera completamente diferente, en el interrogatorio a Doña Lupe, el teniente Silva "se burla", "repite" si no consigue respuesta, "interrumpe" a Doña Lupe, habla "con voz acaramelada", "la reprende", "le habla francamente", "ordena", etc. Por su parte la interrogada se siente intimidada: "murmura con los dientes chocándole", "balbucea", "gime", "aúlla" y "tiembla".

En segundo lugar, el habla popular está cargada de peruanismos y se inclina a usar expresiones que se refieren al ámbito de lo sexual, des-

De paso sea recordado que al mezclar historia policial con historia de amor Vargas Llosa comete un grave pecado contra "el tercer 'mandamiento' de las reglas literarias de van Dine: 'No puede existir ninguna historia amorosa. La misión consiste en llevar a un criminal al juzgado y no a una pareja amorosa al altar'" ( Badenberg/ Honold/ Horstmann 284). 
tacándose el lenguaje del teniente Silva por su "machismo lingüístico" (298) y su vulgaridad (cfr. la familia de palabras en torno al 'huevo', a la 'chucha' y al verbo 'calatearse').

Por otro lado hay que constatar que los personajes pertenecientes al grupo social del poder, como, por ejemplo, los miembros superiores de la Base Aérea, casi nunca emplean expresiones familiares, vulgares o peruanismos.

\section{Conclusión}

Se puede concluir que Vargas Llosa nos presenta en su novela policial ¿Quién mató a Palomino Molero? un 'modelo' policial doblemente híbrido: en primer lugar, por contener tanto elementos de la novela de enigma (p.e. deducciones, abducciones, misterio, pareja clásica de investigadores, aclaración del crimen) como de la novela negra (violencia, sexo, crítica social, lenguaje duro y popular).? Se trataría, por lo mismo, de una hibridez intragenérica. En segundo lugar, podemos hablar también aquí de una hibridez intergenérica en la medida que Vargas Llosa mezcla en esta novela diferentes géneros literarios como, por ejemplo, el género policial, la novela cortés, la comedia en la tradición realista de Cervantes y, por último, la novela de amor. Además, parece ser por todo lo expuesto una novela, ante todo y predominantemente, moderna (búsqueda de la realidad/verdad, aclaración del crimen) con algunos pocos elementos posmodernos (cierta enigmatización de la realidad al final de la obra, fracaso de la búsqueda en lo social aunque no en lo cognoscitivo).

\section{Bibliografía}

Angvik, Birger.

La narración como exorcismo. Mario Vargas Llosa, obras (1963-2003). Lima: Fondo de Cultura Económica, 2004.

Badenberg, Nana / Honold, Alexander / Horstmann, Susanne. “¿Quién mató a Palomino Molero? Vargas Llosa y la novela policial". Revista de Crítica Literaria Latinoamericana, XV, 30 (2 sem. de 1989): 277316.

7 Por eso, con Badenberg/Honold/ Horstmann se puede decir que nuestro escritor peruano jugó en esta novela con las reglas estrictas de la novela policial clásica pero respetándolas a la vez" (cfr. 311). 
Brown, James W.

"El síndrome del expatriado: Mario Vargas Llosa y el racismo peruano". José Miguel Oviedo (ed.). Mario Vargas Llosa. Madrid: Taurus, 1981. 15-24.

Castro-Klarén, Sara. $\quad$ Mario Vargas Llosa: Análisis introductorio. Lima: Latinoamericana Editores, 1988.

Gallagher, D. P. Modern Latin American Literature. Londres-Oxford-Nueva York: Oxford University Press, 1973.

Kloepfer, Rolf / Zimmermann, Klaus.

"Mario Vargas Llosa". Wolfgang Eitel (ed.). Lateinamerikanische Literatur der Gegenwart. In Einzeldarstellungen. Stuttgart: Kröner, 1978. 469493.

Kristal, Efraín. Temptation of the World. The Novels of Mario Vargas Llosa. Nashville: Vanderbilt University Press, 1998.

Oviedo, José Miguel. Mario Vargas Llosa: la invención de una realidad. 2a ed. Barcelona: Barral, 1977.

"Tema del traidor y del héroe: sobre los intelectuales y los militares en Vargas Llosa". J. M.O. (ed.). Mario Vargas Llosa. Madrid: Taurus, 1981. 47-65.

Osorio Tejada, Nelson. "La expresión de los niveles de realidad en la narrativa de Vargas Llosa". Asedios a Vargas Llosa. Santiago: Editorial Universitaria, S.A., 1972. 67-88.

Scheerer, Thomas. Mario Vargas Llosa. Leben und Werk. Eine Einführung. Francforto del Meno: Suhrkamp, 1989.

Vargas Llosa, Mario. ㄴ. ¿ ¿Quién mató a Palomino Molero? Barcelona: Seix Barral, 1991. 